\title{
Abacavir/lamivudine combination in the treatment of HIV: a review
}

This article was published in the following Dove Press journal:

Therapeutics and Clinical Risk Management

23 February 2010

Number of times this article has been viewed

\section{Geetha Sivasubramanian' Emmanuel Frempong-Manso ${ }^{2}$ Rodger D MacArthur' \\ 'Division of Infectious Diseases, Wayne State University, Detroit, MI, USA; ${ }^{2}$ University of Pittsburgh Medical Center, Shadyside Hospital, Pittsburgh, $\mathrm{OH}$, USA}

Correspondence: Rodger D MacArthur Division of Infectious Diseases, Wayne State University, Detroit, MI, USA $\mathrm{Tel}+$ I 3|3-993-0930

Email rmacarthur@med.wayne.edu

\begin{abstract}
Abacavir has been at the center of research and clinical interest in the last two years. The frequency of the associated abacavir hypersensitivity syndrome has decreased substantially since the introduction of routine testing for the HLA-B*5701 allele; the activity of the drug in HIV-infected persons with HIV RNA values more than 100,000 copies/mL has been questioned; the possible increased risk of myocardial infarction after recent exposure to abacavir has been debated; and the drug has been moved from the "recommended" category to the "alternative" category in several guidelines. Still, the drug remains a useful agent in combination with other drugs, including lamivudine, for the treatment of HIV infection. This review will focus on the pharmacokinetics, activity, side effects, and resistance profile of both abacavir and lamivudine, including a thorough review of all of the recent studies relevant to both drugs.
\end{abstract}

Keywords: HIV, abacavir, lamivudine

\section{Introduction/background}

It has been more than 20 years since the introduction of the first antiretroviral (ARV) drug zidovidine (AZT, ZDV). Early ARV therapy regimens required patients to take many pills per dose multiple times per day, which often led to poor adherence and treatment failure. It is thus of no surprise that in later years, simplified regimens (fixed dose combinations, FDCs) have emerged. Current recommendations suggest starting treatment-naïve patients on 2 nucleoside reverse transcriptase inhibitors (NRTIs) with either a protease inhibitor (PI) or a nonnucleoside reverse transcriptase inhibitor (NNRTI). ${ }^{1}$ One such NRTI combination is abacavir (ABC) and lamivudine (3TC) which is also available as a FDC. This article provides a comprehensive review of these two drugs.

\section{Abacavir (ABC)}

Abacavir is available as a single-agent, in dual combination with lamivudine (Epzicom $^{\circledR}$; ViiV Healthcare), and as a triple combination with zidovudine and lamivudine (Trizivir ${ }^{\circledR}$; ViiV Healthcare). A generic form, abacavir sulfate is also available. The recommended adult dose of $\mathrm{ABC}$ is $300 \mathrm{mg}$ twice daily or $600 \mathrm{mg}$ once daily. No dose adjustment is required for renal dysfunction. ${ }^{1}$

\section{Chemistry and pharmacodynamics}

Abacavir is a synthetic carbocyclic nucleoside analogue of the purine, guanine. It is converted to the active metabolite carbovir triphosphate (CBV-TP) through a three-step phosphorylation and deamination process by intracellular enzymes. The active metabolite 
CBV-TP is an analog of deoxyguanosine-5-triphospahte (dGTP), which competes with the endogenous dGTP and blocks its incorporation into the HIV viral DNA. This results in termination of chain elongation and inhibition of viral replication.

\section{Pharmacokinetics}

Abacavir is rapidly absorbed and is widely distributed. It has good bioavailability, (approximately 83\%). Abacavir has significant penetration into cerebral spinal fluid (CSF) $)^{2,3}$ and has been recovered in the semen. ${ }^{4}$ Mean time to peak after single or multiple oral dosages is 0.7 to 1.7 hours. Approximately $50 \%$ of the drug binds to human plasma proteins. The primary route of elimination of $\mathrm{ABC}$ is metabolism by the two hepatic enzymes alcohol dehydrogenase (ADH) and glucuronyl transferase (GT). Alcohol dehydrogenase metabolizes approximately $36 \%$ of the dose to inactive carboxylate and GT metabolises approximately $30 \%$ of the dose to inactive glucuronide metabolite. Although $\mathrm{ABC}$ is metabolized by the liver, it does not inhibit or induce cytochrome P-450 (CYP-450) enzymes and therefore does not interact with medications metabolized by this system. ${ }^{2}$ Eighty-three percent of the dose of the drug is excreted primarily in the urine as metabolites, about $2 \%$ as unchanged drug; $66 \%$ of the dose is excreted as the two major metabolites, the 5'-carboxylate and the $5^{\prime}$ '-glucuronide. ${ }^{2}$

The serum half-life of elimination $\left(t_{1 / 2}\right)$ is about 1.5 hours but the drug has been shown in a number of pharmacokinetic and clinical studies to be efficacious when administered once daily. ${ }^{5-9}$ This is because the activity and efficacy of the drug is mainly determined by its intracellular concentration. When ABC is converted intracelluarly to CBV-TP, the phosphorylation process essentially results in entrapment of the active metabolite in the cell leading to higher accumulation of the active agents than is achieved in the serum. Thus, the concentration of the active drug intracelluarly is higher than that measured in the serum. ${ }^{6}$ As the frequency of drug administration is closely related to the pharmacokinetic properties of a drug, the key parameter is the half-life. However, the serum $T_{1 / 2}$ of ABC, as is true of all NRTIs, is of little use in developing a dosing schedule. Rather, it is the intracellular half-life of the nucleoside triphosphate that is the relevant parameter.

Kewn et al showed in a pharmacokinetic study using the $300 \mathrm{mg}$ dose once daily dose in six patients that CBVTP levels remained above the inhibitory levels of the virus after 24 hours. $^{7}$ Using the same method, Harris et al studied CBV-TP levels in peripheral mononuclear cells (PBMCs) of
5 HIV-positive adults taking ABC $600 \mathrm{mg}$ once a daily for 5 to 17 months as a component of multiple drug rescue therapy. It was shown that the half life of CVB-TP was greater than 12 hours. $^{8}$ A slightly larger study than that done by Harris et al was carried out by Piliero et al involving twenty patients given ABC $300 \mathrm{mg}$ dose over a 24-hour period. Results showed a mean PBMC intracellular half-life of 20.64 and mean plasma half-life of 2.59 hours respectively. ${ }^{6}$

Drusano et $\mathrm{al}^{9}$ using an in vitro pharmacodynamic model system, provided further support for once daily dosing. This group examined $\mathrm{ABC}$ dosing and ascertained the impact of the administration schedule on the activity of the drug. The antiviral effect and relationship to pharmacokinetics of a continuous infusion of $\mathrm{ABC}$ over 24 hours were compared with once-daily and twice-daily doses. A similar ABC exposure and antiviral effect was observed in all three regimens.

In an open-label, 2-period, crossover study, 34 HIVinfected male and female subjects stabilized on antiretroviral regimens containing either $\mathrm{ABC} 600 \mathrm{mg}$ onc daily or $\mathrm{ABC}$ $300 \mathrm{mg}$ twice daily by Moyle et $\mathrm{al}^{10}$ the intracellular CBV-TP values were similar, providing further pharmacokinetic support for the interchangeability of these two regimens.

\section{Adverse events}

A significant number of patients have poor adherence to antiretroviral medications secondary to intolerance and adverse effects. The most important adverse event associated with $\mathrm{ABC}$ is the $\mathrm{ABC}$ hypersensitivity reaction (ABC HSR). The ABC HSR is an idiosyncratic multiorgan clinical syndrome usually characterized by a sign or symptom in 2 or more of the following groups: (i) fever; (ii) rash; (iii) gastrointestinal: nausea, vomiting, diarrhea or abdominal pain; (iv) constitutional: generalized malaise, fatigue or achiness; and (v) respiratory: dyspnea, cough, and/or pharyngitis. ABC HSR can be overlooked, as these symptoms may mimic various other disease conditions such as influenza, pneumonia, gastroenteritis. At one center, $15 \mathrm{HIV}$-positive patients who had symptoms of ABC HSR were compared with $30 \mathrm{HIV}$-positive patients who had symptoms of influenza. ${ }^{11}$ Gastrointestinal symptoms occurred in $60 \%$ of patients with ABC HSR versus $6 \%$ of patients with influenza. Although fever and myalgia were commonly observed in both groups, rash occurred in $47 \%$ of the patients with ABC HSR and in only $6 \%$ of patients with influenza. Although demographic characteristics, vital signs, and laboratory tests did not differ between the groups, respiratory symptoms that occurred without gastrointestinal symptoms were much more likely to be caused by influenza than by ABC HSR. 
The overall incidence rate of ABC HSR is approximately $8 \%$ with some variability depending on genetic susceptibility of the population. ${ }^{12}$ More than $93 \%$ of ABC HSR reactions occur during the first 6 weeks of treatment. ${ }^{12-17}$ The median time to develop the reaction is 8 days. The reaction can develop on the first day of receipt of $A B C$ therapy and has been reported to occur up to 160 days after initiation. Patients with a history of the $A B C$ HSR who have been rechallenged with the drug have experienced unanticipated life-threatening consequences. Among 112 patients with ABC HSR who were rechallenged, an anaphylactic or immediate type of hypersensitivity reaction occurred in $20 \% .^{15}$ Therefore, patients with clinically suspected ABC HSR should not be rechallenged. There have been case reports of patients who developed the $\mathrm{ABC}$ HSR after re-initiation of $\mathrm{ABC}$ after a hiatus in taking the medication, but this occurrence is believed to be rare. ${ }^{16}$ Interestingly, these patients had no symptoms of HSR during the first period of $\mathrm{ABC}$ administration. A study of 145 patients who interrupted $A B C$ treatment showed 1 case of hypersensitivity but no statistical difference in incidence between the study patients who did not interrupt $\mathrm{ABC} .^{17}$

The ABC HSR is a clinical diagnosis. Physical findings associated with the ABC HSR in some patients include lymphadenopathy, mucous membrane lesions (conjunctivitis and mouth ulcerations), and rash. The rash usually is maculopapular or urticarial, but may be variable in appearance. ${ }^{18}$ Abnormal laboratory findings may occur, but they are no means specific or diagnostic. Eosinophilia usually is not present. Leukopenia, lymphopenia and thrombocytopenia may all occur. ${ }^{18}$ Approximately $10 \%-15 \%$ of cases have elevated liver enzymes and a rise in creatinine phosphokinase levels also has been reported. ${ }^{19}$ Other abnormal laboratory values noted include elevations in alkaline phosphatase, blood urea nitrogen, serum creatinine, and lactic dehydrogenase levels. Disseminated intravascular coagulation has also been reported as a manifestation of ABC HSR. ${ }^{20}$

A fever that develops within a few weeks after the initiation of therapy with abacavir may be due to causes other than hypersensitivity. Most common is the likelihood that simultaneous initiation of treatment with drugs, such as trimethoprim-sulfamethoxazole, efavirenz, or nevirapine, may be the cause. The incidence of adverse reactions, including hypersensitivity, to each of these drugs is greater than that of ABC. ${ }^{21-23}$ The presence of gastrointestinal or respiratory symptoms that accompany a rash or fever suggest ABC HSR as the likely cause. Opportunistic infections can manifest shortly after initiation of ARV as a result of immune reconstitution and should also be in the differential diagnosis.

Altered or unusual drug metabolism and a susceptible immune system are believed to be important cofactors for the development of drug-related hypersensitivity. ${ }^{24} \mathrm{HIV}$-infected persons are likely to be at special risk of developing hypersensitivity reactions because of the disease associated perturbations in the immune system. ${ }^{25}$ Two members of a family who were treated with $\mathrm{ABC}$ both developed the HSR suggesting a genetic component to $\mathrm{ABC}$ HSR ${ }^{26}$ Multiple studies have shown an association of the ABC HSR with HLA-B*5701. ${ }^{27-29}$ The use of this screening test to identify high risk patients has been shown by Rauch et al to reduce the occurrence of the ABC HSR. ${ }^{30}$ In this cohort involving more than 260 patients, the authors observed that the use of HLA-B*5701 screening decreased ABC HSR from $7 \%$ to $<1 \%$. Similarly, the incidence of the ABC HSR decreased from $6.2 \%$ to $0.5 \%$ in a UK cohort of 185 patients treated with $\mathrm{ABC}$ using HLA screening. ${ }^{31}$

The PREDICT-1 trial, a double-blind, prospective, randomized study involved 1956 patients from 19 countries, who were infected with human immunodeficiency virus type 1 and who had not previously received abacavir evaluated the effectiveness of prospective HLA-B*5701 screening to prevent the hypersensitivity reaction to abacavir. The study found a significantly lower incidence of ABC HSR in the prospective-screening group (3.4\%) than in the control group (7.8\%), $(P<0.001) .{ }^{32}$

Similar results were obtained from the SHAPE study further strengthening the utility of prescreening with the HLA-B*5701 test prior to starting abacavir. ${ }^{33}$

On the basis of these studies, the 2008 DHHS guidelines panel recommends screening for HLA-B $* 5701$ before starting patients on an abacavir-containing regimen, to reduce the risk of hypersensitivity reaction (AI recommendation). ${ }^{1}$

In an analysis of 5,332 patients enrolled in $\mathrm{ABC}$ clinical trials, being ART-experienced at the start of $\mathrm{ABC}$ therapy and being of African descent were associated with a nearly $40 \%$ reduction in the risk of hypersensitivity. ${ }^{19}$ Patients of white race were found to be at significantly greater risk in another study of a population with a low percentage of ethnic minorities. ${ }^{14}$ Accumulated data suggest that factors that predict a lower likelihood to develop ABC HSR are: male sex, African racial origin, more advanced disease, and being an antiretroviral-experienced patient before initiation of $\mathrm{ABC} \cdot{ }^{12,14}$

There are no established interventions that will preempt the development of the ABC HSR. Patients randomized to receive prednisone $40 \mathrm{mg} /$ day for the first 2 weeks when initiating $\mathrm{ABC}$ therapy in combination with nevirapine (NVP) and ZDV/3TC experienced a slightly higher incidence of hypersensitivity than those patients who did not receive prednisone (17\% versus $10 \%$, respectively). ${ }^{34}$ 
The decision whether to stop $\mathrm{ABC}$ therapy or to cautiously continue it is an important one when ABC HSR cannot clinically be excluded. For instance, a rash may occur during $\mathrm{ABC}$ therapy without HSR. $\mathrm{ABC}$ may be an important component of an antiretroviral regimen that may be difficult to stop unless absolutely necessary because other alternatives may be lacking. However, the potential severity of $A B C$ hypersensitivity calls for prudence. The overall case fatality rate is estimated to be $0.03 \% .{ }^{19} \mathrm{ABC}$ should be discontinued if the timing, severity, and combination of clinical symptoms are suggestive. When it is not clear if clinical symptoms are due to the ABC HSR, the drug may be continued under close clinical surveillance. For patients requiring hospitilzation due to ABC HSR, treatment is supportive.

In a recent large, prospective observational cohort of HIV infected individuals by the Data Collection on Adverse Events of Anti-HIV Drugs (DAD), the risk of myocardial infarction in relation to exposure to NRTI's was studied. ${ }^{35}$ DAD is an international collaboration of 11 cohorts, following 33,347 HIV-1-infected individuals at 212 clinics in Europe, the US, and Australia. ${ }^{6,723}$ All participants were under active follow-up in their cohorts at the time of enrolment into DAD (December 1999 to January 2005). The study used Poisson regression models to quantify the relation between cumulative, recent (currently or within the preceding 6 months), and past use of zidovudine, didanosine, stavudine, lamivudine, and abacavir and development of myocardial infarction. The study found no associations between the rate of myocardial infarction and cumulative or recent use of zidovudine, stavudine, or lamivudine. However recent use of abacavir or didanosine was associated with an increased rate of myocardial infarction (compared with those with no recent use of the drugs, relative rate $1.90,95 \%$ confidence interval [CI 1.47 to $2.45[P=0.0001]$ with abacavir and 1.49 , 1.14 to $1.95[P=0.003]$ with didanosine). After adjustment for predicted 10-year risk of coronary heart disease, recent use of both didanosine and abacavir remained associated with increased rates of myocardial infarction $(1.49,1.14$ to 1.95 $[P=0.004]$ with didanosine; $1.89,1.47$ to $2.45[P=0.0001]$ with abacavir). It was concluded that an increased risk of myocardial infarction exists in patients exposed to abacavir and didanosine within the preceding 6 months. This excess risk was not explained by underlying established cardiovascular risk factors and was not present beyond 6 months after drug cessation.

The reproducibility of this finding in a different (but somewhat DAD-overlapping) dataset was explored and plausible biological mechanisms were sought by using the
SMART study data. Biomarkers, ischemic changes on the electrocardiogram, and rates of various predefined types of cardiovascular disease (CVD) events according to NRTIs used were explored in the Strategies for Management of Anti-Retroviral Therapy (SMART) study. ${ }^{36}$ Patients receiving abacavir and not didanosine were compared with those receiving didanosine, and to those receiving NRTIs other than abacavir or didanosine (other NRTIs). Current use of abacavir was associated with an excess risk of CVD compared with other NRTIs. Adjusted hazard ratios for clinical myocardial infarction ( $\mathrm{n}=19)$, major CVD (myocardial infarction [MI], stroke, surgery for coronary artery disease, and CVD death $\mathrm{n}=70$ ); expanded CVD (major CVD plus congestive heart failure, peripheral vascular disease, coronary artery disease requiring drug treatment, and unwitnessed deaths $\mathrm{n}=112$ ); were 4.3 [ $95 \% \mathrm{CI} 1.4$ to 13.0 ], 1.8 (1.0 to 3.1), and 1.9 (1.3 to 2.9). At baseline in a subset of patients with biomarker data, high sensitivity-C-reactive protein and interleukin-6 were $27 \%(P=0.02)$ and $16 \%(P=0.02)$ higher for patients receiving abacavir $(\mathrm{N}=175)$ compared with those receiving other NRTIs $(\mathrm{N}=500)$. The analysis concluded that abacavir was associated with an increased risk of CVD and may cause vascular inflammation, which may precipitate a CVD event.

Analysis was performed by GlaxoSmithKline of GlaxoSmithKline-sponsored clinical trials with $\geq 24$ weeks of combination antiretroviral therapy comprising 14,174 HIVinfected adults who received ABC $(n=9502 ; 7641$ personyears) or not ( $n=4672 ; 4267$ person-years). The baseline demographics and HIV disease characteristics, including lipids and glucose values, were similar. MI rates were comparable among subjects exposed $[\mathrm{n}=16(0.168 \%$; CI 0.096 to $0.273 ; 2.09$ per 1000 person-years $)]$ or not $[n=11(0.235 \%$; CI 0.118 to $0.421 ; 2.57$ per 1000 person-years)] to $\mathrm{ABC}$ containing therapy. In this analysis there were few MI events overall and no excess risk of MI with $\mathrm{ABC}$ therapy. ${ }^{37}$

A study conducted by Veterans Affairs investigators, involving 19,424 people with an average 3.9 years of follow-up, showed a slight, statistically non-significant increase in MI with abacavir. ${ }^{38}$ The association of $\mathrm{ABC}$ use with AMI was much weaker after adjusting for traditional cardiovascular risk factors, including chronic kidney disease (CKD). Predictably, a significantly higher number of patients with estimated glomerular filtration rate below 60 received abacavir compared to tenofovir $(P<0.0001)$, which has been associated with acute kidney injury and worsening renal function.

We have to keep the possibility of channeling bias in interpreting the DAD study results, since patients with CKD are more likely to be prescribed abacavir than tenofovir and 
CKD is a risk factor for $\mathrm{MI}$, and the DAD study did not adjust for CKD. In addition, other studies, such as the AIDS Clinical Trials Group (ACTG) ALLRT observational cohort did not find an association of abacavir use with increased risk for myocardial infarction. ${ }^{39}$

Although there have been conflicting results in these studies, abacavir/lamivudine combination has now been moved to the alternative first line treatment category in treatment naïve patients. Pending further studies, we should exercise caution in using abacavir in treating HIV-infected patients with underlying high risk for cardiovascular disease.

\section{Lamivudine (3TC)}

Lamivudine is available as a single agent, in dual combination with abacavir (ABC/3TC or epzicom), and zidovudine (AZT/3TC or combivir), and in triple combination with zidovudine and abacavir (ABC/AZT/3TC or trizivir). The recommended adult dosage is $150 \mathrm{mg}$ twice daily or $300 \mathrm{mg}$ once daily orally in combination with other antiretroviral agents. The recommended dose in renal insufficiency (creatinine clearance 30 to $50 \mathrm{~mL} / \mathrm{min}$ ) is $150 \mathrm{mg}$ daily. For creatinine clearance 15 to $29 \mathrm{~mL} / \mathrm{min}$, the dose is $100 \mathrm{mg}$ daily, following a loading dose of $150 \mathrm{mg}$; for creatinine clearance 5 to $14 \mathrm{~mL} / \mathrm{min}$, the dose is $50 \mathrm{mg}$ daily, following a loading dose of $150 \mathrm{mg}$; for creatinine clearance $<5 \mathrm{~mL} / \mathrm{min}$, the dose is $25 \mathrm{mg}$ daily, following a loading dose of $50 \mathrm{mg}$. ${ }^{1}$ These dosing adjustments are based on achieving levels comparable to those in persons without renal impairment.

\section{Chemistry and pharmacodynamics}

Lamivudine (3TC) is a cytosine analogue. It undergoes 3-step phosphorylation like $\mathrm{ABC}$ to form the active metabolite lamivudine triphosphate (3TC-TP) which competes with endogenous cytosine. In the first step, deoxycytidine kinase catalyzes the formation of the monophosphate. In the second step, the monophosphate is phosphorylated by cytidine monophosphate (deoxycytidine monophosphate kinase) to the diphosphate. Finally, the diphosphate is converted to the triphosphate by the enzyme nucleoside diphosphate kinase. After 3TC is triphosphorylated, the principle mode of action is inhibition of HIV reverse transcription via viral DNA chain termination. It should be pointed out that the phosphorylation pathways for $3 \mathrm{TC}$ and $\mathrm{ABC}$ are different.

\section{Pharmacokinetics}

$3 \mathrm{TC}$ is rapidly absorbed after oral administration. The absolute bioavailability is approximately $82 \%$ in adults. The drug reaches a maximum concentration of 0.5 to 1.5 hours after oral administration. Less than $36 \%$ of the drug binds to plasma protein. The half life of elimination is 5 to 7 hours in adults but it is suitable to be administered as once daily dose as discussed in the next paragraph. Lamivudine is excreted primarily in the urine as unchanged drug.

In a study comparing the plasma pharmacokinetics of $3 \mathrm{TC}$ administered $150 \mathrm{mg}$ twice daily and $300 \mathrm{mg}$ once daily orally in 13 HIV-1 infected patients, Bruno et al were able to show that although there was statistically significant differences $(P<0.05)$ between the 2 schedules for $\mathrm{C}_{\max }$ and $\mathrm{C}_{\text {min }}$ values, average concentration over the dosage interval and AUC over 2 dosage intervals ( 24 hours) were similar. ${ }^{40}$ The investigators concluded that their results provided the pharmacokinetic basis for using 3TC in a once daily regimen. Similar to ABC, with lamivudine, it is the intracellular concentration of the active metabolite that determines its activity, efficacy and suitability for once daily dosing.

Moore et al evaluated the pharmacokinetics of 3TC phosphorylation in peripheral blood mononuclear cells from 10 asymptomatic, antiretroviral-experienced HIV-1 infected patients who were receiving treatment with a regimen of $3 \mathrm{TC}$ and zidovudine (ZDV). ${ }^{41}$ The patients were randomly assigned to receive 3 TC $150 \mathrm{mg}$ twice a day or $300 \mathrm{mg}$ twice a day for 14 days. The median half-life of intracellular 3TCTP was 15.3 hours for the 150-mg dose and 16.1 hours for the 300-mg dose, a nonsignificant difference. Yuen et $\mathrm{al}^{42}$ compared the steady-state pharmacokinetics of 3TC in plasma and the active metabolite 3TC-TP in PBMCs. In this study, 60 healthy subjects received 3TC $300 \mathrm{mg}$ once daily for 7 days and $150 \mathrm{mg}$ twice daily for 7 days. Steady-state plasma 3TC pharmacokinetics following the once- and twicedaily regimens were bioequivalent with respect to both the AUC from 0 to 24 hours at steady state and average plasma $3 \mathrm{TC}$ concentration at steady state over the dosing interval. Steady-state intracellular 3TC-TP pharmacokinetics after the once- and twice-daily regimens are bioequivalent with respect to AUC and average plasma concentrations, as well as maximal 3TC concentrations. Overall, the results of this study suggest that for key pharmacokinetic parameters, 3TC $300 \mathrm{mg}$ once daily is equivalent to $3 \mathrm{TC} 150 \mathrm{mg}$ twice daily. A summary of the pharmacokinetic properties of $\mathrm{ABC}$ and $3 \mathrm{TC}$ is as shown in Table 1.

\section{Drug interactions}

The combination of 3TC and ABC as an NRTI backbone is ideal and effective because there is no intracellular competition for phosphorylation of these drugs. Furthermore, 3TC pharmacokinetics are not significantly affected by ABC. ${ }^{17}$ 
Table I Pharmacokinetics of abacavir (ABC) and lamividine (3TC) in adults

\begin{tabular}{lll}
\hline Pharmacokinetic property & ABC & 3TC \\
\hline Oral bioavailability (\%) & 83 & $82-86$ \\
$\mathrm{C}_{\max }(\mu \mathrm{g} / \mathrm{ml})$ & 4.26 & 2.04 \\
Mean time to $\mathrm{C}_{\max }$ (hours) & $0.7-1.7$ & $0.5-1.5$ \\
$\mathrm{AUC}(\mu \mathrm{g} * \mathrm{~h} / \mathrm{mL})$ & 11.95 & 8.87 \\
Plasma protein binding $(1 \%)$ & 50 & $<36$ \\
Plasma $T_{1 / 2}$ (hours) & 1.5 & $5-7$ \\
Intracellular $\mathrm{T}_{1 / 2}$ (hours) & 20.64 & $16-19$ \\
Metabolism & Predominantly hepatic metabolism and then & $5 \%-10 \%$ hepatic metabolism; predominantly \\
& renal excretion (about 83\%); 2\% of the drug & renal excretion as unchanged drug via \\
\hline
\end{tabular}

Due to low protein binding, drug-drug interactions are infrequent. As a class, the NRTIs are predominantly excreted by the renal tubular system and interactions with drugs metabolized primarily by the cytochrome P-450 system are not encountered frequently. However drugs influencing renal clearance or intracellular phosphorylation may cause interactions with all of the NRTIs.

The main interaction of $3 \mathrm{TC}$ and other drugs occurs with drugs that use renal tubular excretion for their elimination. In a randomized 2-way cross over study, ${ }^{43} 14 \mathrm{HIV}$-positive patients were given a $300 \mathrm{mg}$ single dose 3TC and trimethoprim/ sulfamethoxazole (TMP/SMX) 160/800 mg for 5 days. It was noted that the area under the curve (AUC) of 3TC increased by $43 \%$, and renal clearance of $3 \mathrm{TC}$ decreased by $30 \%$. This increase was considered not significant, especially given the favorable safety profile of lamivudine. No significant change in any pharmacokinetic parameter occurred for TMP/SMX. Thus, no 3TC dosage adjustment is needed when used with TMP/SMX.

Lamivudine and zalcitabine (ddC) may inhibit the intracellular phosphorylation of one another and therefore such combination in any drug regimen should be avoided. ${ }^{18}$ In vitro data indicate that ribavirin reduces phosphorylation of $3 \mathrm{TC}$, stavudine (d4T) and AZT. However, no pharmacokinetic or pharmacodynamic interaction was observed when ribavirin and 3TC $(n=18)$, d4T $(n=10)$, or AZT $(n=6)$ were coadministered as part of a multi-drug regimen to $\mathrm{HIV} / \mathrm{HCV}$ co-infected patients. ${ }^{18}$

There have been concerns about possible pharmacokinetic interaction between $\mathrm{ABC}$ and ethanol, as both are metabolized by alcohol dehydrogenase. However, in an open labeled randomized, 3- way crossover, phase 1 study, $24 \mathrm{HIV}$ positive males were given alcohol $0.7 \mathrm{~g} / \mathrm{kg}$ and a single dose of $600 \mathrm{mg} \mathrm{ABC}$. It was shown that ABC AUC increased by $41 \%$, half-life of elimination increased by $26 \%$, and plasma maximum concentration increased by $15 \%$. These changes were not considered statistically significant. ${ }^{18,44}$

In a study of $11 \mathrm{HIV}$ infected patients receiving methadone-maintenance therapy (40 $\mathrm{mg}$ and $90 \mathrm{mg}$ daily) with $600 \mathrm{mg}$ of $\mathrm{ABC}$ twice daily (twice the currently recommended dose), oral methadone clearance increased by $22 \%(90 \% \mathrm{Cl}=6 \%-42 \%)$. This alteration will not result in a methadone dose modification in the majority of patients; however, an increased methadone dose may be required in a small number of patients. ${ }^{18}$ Lamivudine does not affect the clearance of methadone.

Both lamivudine and abacavir are listed as Pregnancy Category C drugs. No well-controlled trials of the FDC of abacavir + lamivudine $\left(\right.$ Kivexa $^{\circledR} /$ Epzicom $\left.^{\circledR}\right)$ have been conducted in pregnant women. The drugs should be used in pregnancy only when the benefits outweigh the risks. ${ }^{18}$

\section{Clinical efficacy studies} $\mathrm{ABC} / 3 \mathrm{TC}$ combination in comparison

\section{to other nucleotide combinations}

CNA30024 study, ${ }^{45}$ a multicenter, randomized, doubleblind trial compared the efficacy and safety of ABC/3TC versus AZT/3TC combined with efavirenz (EFV) in 649 antiretroviral-naive HIV-infected patients. Baseline median HIV-1 RNA level was $4.79 \log _{10}$ copies/mL (39\% of patients had HIV-1 RNA levels $>100,000$ copies/mL). Baseline median CD4+ cell count was 264 cells $/ \mu \mathrm{L}$. Participants were randomized to receive either a 300-mg tablet of ABC and a placebo tablet, both administered twice daily, or a 300-mg tablet of AZT and a placebo tablet, both administered twice daily. All participants received a 150-mg tablet of 3TC twice daily plus $600 \mathrm{mg}$ of EFV once daily. The primary objective was a comparison of the proportion of patients achieving plasma HIV-1 RNA levels (VL) $\leq 50$ copies/mL through 
week 48 of the study. At week $48,70 \%$ of patients in the ABC group, compared with $69 \%$ in the AZT group, maintained a confirmed VL of $\leq 50$ copies $/ \mathrm{mL}$ (in the ITT exposed population). There was a significantly different $\mathrm{CD} 4+$ cell increase from baseline at 48 week: 209 cells $/ \mathrm{mm}^{3}$ in the ABC group versus 155 cells $/ \mathrm{mm}^{3}$ in the AZT group $(P=0.005)$. Although both groups had similar baseline CD4+ cell counts, the $\mathrm{ABC}$ group showed a better median absolute $\mathrm{CD} 4+$ cell response at all time points observed. The most common reported adverse effect in the AZT arm was nausea (37\% versus $23 \%$ in the $\mathrm{ABC}$ arm). Reasons for discontinuation of drug in the $\mathrm{ABC}$ arm included nausea (2\%), dizziness (1\%), rash (3\%) and suspected ABC HSR (8\%); in the AZT arm, nausea, dizziness, rash (3\% each) and anemia (4\%) were the most common reasons for drug discontinuation.

The NRTI sub study of the FIRST study ${ }^{46}$ randomized 182 antiretroviral-naïve participants to receive abacavir + lamivudine versus didanosine + stavudine, each combined with a PI, an NNRTI, or both. Mean HIV RNA and CD4+ cell counts at baseline were $5.1 \log _{10}$ copies $/ \mathrm{mL}$ and 212 cells $/ \mathrm{mm}^{3}$. After a median follow-up of 28 months, there was no difference in rates of HIV RNA $>50$ copies $/ \mathrm{mL}$ between the two NRTI groups. However, there was a trend $(0.05 \geq P<0.10)$ for the abacavir + lamivudine group to be better than the didanosine + stavudine group for HIV RNA decreases, CD4+ cell count increases, and tolerability. The combination of didanosine + stavudine is no longer recommended, due to excessive toxicity observed in multiple clinical trials. ${ }^{1}$

A5202 was a phase IIIB, randomized 4-arm study for treatment-naïve subjects of double-blind $\mathrm{ABC} / 3 \mathrm{TC}$ vs TDF/ FTC with open-label efavirenz or atazanavir + ritonavir, stratified by screening HIV RNA $(<$ vs $>100,000$ copies $/ \mathrm{mL}){ }^{47}$ The primary endpoints were time to virologic failure (VF) (confirmed HIV RNA $<1000$ copies/mL at 16 to 24 weeks or 200 cells $/ \mathrm{mL}$ at 24 weeks) and time to first Grade 3/4 adverse event (AE). A5202 enrolled 1858 eligible subjects; 797 had screening HIV RNA $>100,000$ copies/mL. Median follow-up was 60 weeks. $85 \%$ were men, $26 \%$ Black, $25 \%$ Hispanic; mean baseline HIV RNA $=5.1 \log _{10}$ copies $/ \mathrm{mL}$, CD4 $=181 / \mathrm{mm}^{3}$. Among participants with entry HIV $\mathrm{RNA} \geq 100,000$ copies $/ \mathrm{mL}$, time to VF was significantly shorter in the ABC/3TC than TDF/FTC arm $(\mathrm{HR}=2.33$, $95 \%$ CI 1.46 to $3.72, P=0.0003$ ), occurring in 57 and 26 subjects respectively. In a secondary cross-sectional analysis (prior VF and regimen changes included), the proportion (95\% CI) with HIV RNA $<50$ copies $/ \mathrm{mL}$ at week 48 was $75 \%(69 \%$ to $80 \%)$ for $\mathrm{ABC} / 3 \mathrm{TC}$ and $80 \%$ (74\% to $85 \%)$ for TDF/FTC $(P=0.20)$. Subjects receiving ABC/3TC had a shorter time to grade $3 / 4 \mathrm{AEs}(\mathrm{HR}=1.87,95 \% \mathrm{CI} 1.43$ to 2.43, $P<0.0001)$, predominantly general body aches and lipid increases. The comparisons of blinded NRTIs in the lower HIV RNA stratum and each regimen's third drug in both strata are ongoing. Data safety monitoring board review prompted unblinding of NRTIs, additional analyses and recommendations to continue with NRTIs of choice due to the significant virologic efficacy differences by NRTIs for subjects with screening HIV RNA $>100,000$ copies $/ \mathrm{mL}$.

The HEAT study was the first completed, randomized clinical trial to directly compare the efficacy, safety, and tolerability of these agents, each in combination with lopinavir/ ritonavir in antiretroviral-naive patients. Six hundred and eighty-eight antiretroviral-naive, HIV-1-infected patients were randomized in this double-blind, placebo-matched, multicenter, non-inferiority study to receive a once-daily regimen of either ABC/3TC $600 \mathrm{mg} / 300 \mathrm{mg}$ or TDF/FTC $300 \mathrm{mg} / 200 \mathrm{mg}$, both with lopinavir/ritonavir $800 \mathrm{mg} / 200 \mathrm{mg}$. Primary endpoints were the proportion of patients with HIV-1 RNA below 50 copies $/ \mathrm{mL}$ at week 48 (missing = failure, switch included analysis) and the proportion of patients experiencing adverse events over 96 weeks. At week $48,68 \%$ in the $\mathrm{ABC} / 3 \mathrm{TC}$ group vs. $67 \%$ in the TDF/FTC group achieved an HIV-1 RNA below 50 copies $/ \mathrm{mL}$ (intent-to-treat exposed missing = failure, $95 \%$ confidence interval on the difference -6.63 to 7.40 , $P=0.913$ ), demonstrating the non-inferiority of $\mathrm{ABC} / 3 \mathrm{TC}$ to TDF/FTC at week 48. Non-inferiority of the two regimens was sustained at week 96 (60\% versus $58 \%$, respectively, $95 \%$ confidence interval -5.41 to $9.32, P=0.603$ ). In addition, efficacy of both regimens was similar in patients with baseline HIV-1 RNA $\geq 100,000$ copies/mL or CD4 cell counts below 50 cells $/ \mu \mathrm{L}$. In this analysis, both $\mathrm{ABC} / 3 \mathrm{TC}$ and TDF/FTC provided comparable antiviral efficacy, safety, and tolerability when each was combined with lopinavir/ritonavir in treatmentnaive patients. ${ }^{48}$

Only 2 studies are available comparing the $\mathrm{ABC} / 3 \mathrm{TC}$ and TDF/FTC combinations head-on, with 1 (A5202) still not completed. Conflicting results were noted between both studies in terms of virologic failure and pending further studies, caution should be exercised in using abacavir/lamivudine in treatment naïve patients with baseline RNA $>100,000 \mathrm{~cm}^{3} / \mathrm{mL}$.

\section{ABC/3TC as separate agents combined with a PI or NNRTI}

The NEAT trial ${ }^{49}$ was an international, multicenter, randomized, open-label study that compared the efficacy, durability, and tolerability of unboosted fos-amprenavir (f-AMV) 
$1400 \mathrm{mg}$ twice daily, with nelfinavir (NFV) $1250 \mathrm{mg}$ twice daily, in antiretroviral therapy (ART)-naive HIV-infected adults with plasma viral load at screening greater than or equal to 5000 copies/mL. Patients were randomly assigned to f-AMV or NFV $(2: 1)$ for a minimum of 48 weeks, with a background of $\mathrm{ABC}$ and 3TC. A total of 166 patients were on the f-AMV arm and 83 on the NFV arm. Most of the patients in the study had a previous CDC class $\mathrm{C}$ event. At the time of enrollment, the median HIV RNA level was 4.82 to $4.85 \log _{10}$ copies/mL and median CD4+ count was 212 to 214 cells $/ \mathrm{mm}^{3}$. After 48 weeks of study, favorable virologic and immunologic responses were observed for both groups. The ABC/3TC backbone combined with either f-AMV or NFV was well- tolerated.

The SOLO study ${ }^{50}$ compared the magnitude and durability of the antiviral response to ritonavir-boosted fos-amprenavir (f-AMV/r) with NFV, each administered with ABC and 3TC. This was a randomized, open label-study in antiretroviral therapy-naïve advanced HIV+ patients. Three hundred and twenty patients received f-AMV/r (1400/200 mg) once daily and 327 patients received NFV $1250 \mathrm{mg}$ twice daily; both groups received the $\mathrm{ABC} / 3 \mathrm{TC}$ backbone given twice daily. Median CD4+ cell count was 170 cells $/ \mathrm{mm}^{3}$ and median HIV RNA level was $4.78 \log _{10}$ copies/mL. At week 48, the $\mathrm{f}-\mathrm{AMV} / \mathrm{r}$ arm was noninferior to the NFV arm. Sixty-nine percent of patients in the $\mathrm{f}-\mathrm{AMV} / \mathrm{r}$ group and $68 \%$ in the NFV group had HIV RNA levels $<400$ copies $/ \mathrm{mL} ; 55 \%$ of patients in the $\mathrm{f}-\mathrm{AMV} / \mathrm{r}$ group and $53 \%$ in the NFV group had HIV RNA levels $<50$ copies/mL. Diarrhea was the only adverse event to be statistically significantly different in incidence between treatment groups $(16 \%$ for NFV versus $9 \%$ for $\mathrm{f}-\mathrm{AMV} / \mathrm{r} ; P=0.008)$.

The ZODIAC study ${ }^{51}$ evaluated the efficacy and safety of once daily versus twice daily doses of ABC. This was a randomized double-blind study. Patients received $600 \mathrm{mg}$ once daily $(\mathrm{n}=384)$ versus $300 \mathrm{mg}$ administered twice daily ( $\mathrm{n}=386$ ) in combination with $300 \mathrm{mg}$ 3TC and $600 \mathrm{mg}$ of EFV each administered once daily in antiretroviral-naive patients over 48 weeks. The baseline median plasma HIV-1 RNA level was $4.89 \log 10$ copies/mL ( $44 \%$ of the patients had viral load $>100,000$ copies/mL at baseline); median CD4+ cell count was 262 cells $/ \mathrm{mm}^{3}$. ABC administered once daily was non-inferior to the twice-daily regimen, with $66 \%$ and $68 \%$ of patients in these respective treatment arms achieving a confirmed plasma HIV-1 RNA level $<50$ copies/mL at 48 weeks. The ABC once-daily and twice-daily regimens were similar with respect to infrequency of virologic failure (10\% vs $8 \%$ ), emergence of resistance mutations, CD4+ cell count increases from baseline, safety profile, and incidence of ABC-related hypersensitivity reactions (9\% versus $7 \%$ ).

The CLASS study ${ }^{52}$ was an open-label, multicenter, randomized trial of up to 3 consecutive treatment regimens over 96 weeks. Two hundred ninety-one subjects received an ABC/3TC backbone with either efavirenz (NNRTI, $n=97$ ), ritonavir-boosted amprenavir (PI, $\mathrm{n}=96)$, or stavudine (NRTI, $\mathrm{n}=98$ ). Participants receiving boosted amprenavir (APV/r) were later switched to boosted fos-amprenavir (f-AMV/r) to decrease bill burden. At week 96, there were no statistically significant differences between arms in the percentages of subjects with HIV RNA levels $<50$ copies $/ \mathrm{mL}$ by ITT analysis (missing data = failure): $68 \%, 58 \%, 61 \%$ on EFV, APV/r and d4T arms, respectively. The NNRTI arm had a statistically significantly greater percentages of participants with HIV RNA levels $\leq 50$ copies/mL at weeks $24(P=0.018)$ and $48(P=0.047)$. Twenty-one subjects had HSR attributed to ABC (7.3\%). In conclusion, all treatment regimens demonstrated excellent 96-week results. Secondary analyses favored the NNRTI regimen over the PI and NRTI regimens.

\section{$\mathrm{ABC} / 3 \mathrm{TC}$ fixed dose as backbone in studies comparing PI versus NNRTI}

The KLEAN (Kaletra versus Lexiva with Epivir and Abacavir in ART-Naïve patients) study ${ }^{53}$ compared ritonavir-boosted lopinavir (LPV/r) to ritonavir-boosted $\mathrm{f}-\mathrm{AMV} / \mathrm{r}$ combined with an ABC/3TC fixed-dose combination backbone. This was an open-label, non-inferiority study. Eight hundred seventy-eight antiretroviral-naive, HIV-1-infected persons were randomized to receive either f-AMV/r $700 \mathrm{mg} / 100 \mathrm{mg}$ twice daily or LPV/r $400 \mathrm{mg} / 100 \mathrm{mg}$ twice daily, each with ABC/3TC $600 \mathrm{mg} / 300 \mathrm{mg}$ once daily. At week 48, noninferiority of $\mathrm{f}-\mathrm{AMV} / \mathrm{r}$ to $\mathrm{LPV} / \mathrm{r}$ was shown, with $73 \%$ patients in the $\mathrm{f}-\mathrm{AMV} / \mathrm{r}$ group and $71 \%$ in the LPV/r group achieving HIV-1 RNA levels $<400$ copies/mL. Treatment discontinuations due to an adverse event were few and occurred with similar frequency in the two treatment groups. The ABC HSR occurred in $6 \%$ of persons on the $\mathrm{f}-\mathrm{AMV} / \mathrm{r}$ arm versus $4 \%$ on the $\mathrm{LPV} / \mathrm{r}$ arm.

In the ESS30008 study, ${ }^{54} \mathrm{ABC}$ and $3 \mathrm{TC}$ administered twice daily were compared with fixed-dose combination (FDC) ABC/3TC administered once daily, both with either a PI or NNRTI in ARV-experienced persons. Two hundred sixty HIV-infected subjects with more than 6 months of ABC and 3TC administered twice daily plus a PI or NNRTI with an HIV RNA level $<400$ copies/mL for $>3$ months and a CD4+ cell count $>50$ cells $/ \mathrm{mm}^{3}$ were randomized $1: 1$ to continue 
$\mathrm{ABC} / 3 \mathrm{TC}$ administered twice daily or switch to the FDC $\mathrm{ABC} / 3 \mathrm{TC}$ administered once daily. At baseline, median time on $\mathrm{ABC}$ and $3 \mathrm{TC}$ administered twice daily was 22 months, and median CD4+ cell count and HIV RNA level were 554 cells/mm and $<50$ copies/mL, respectively. ABC/3TC FDC administered once daily was established as not inferior to $\mathrm{ABC} / 3 \mathrm{TC}$ administered twice daily based on the proportion of nonvirologic failures. Proportions of persons in each group with an HIV-1 RNA level $<50$ copies $/ \mathrm{mL}$ were $81 \%$ $(\mathrm{ABC} / 3 \mathrm{TC} \mathrm{FDC})$ and $82 \%(\mathrm{ABC}+3 \mathrm{TC} \mathrm{BID})$ at week 48 by ITT $($ missing data $=$ failure $)$. Virologic failure was rare ( 2 patients taking the once-daily regimen, 4 patients taking the twice-daily regimen). There was a low incidence of grade 2 through 4 adverse events and no drug-related serious adverse events or hypersensitivity reactions seen.

The CAL30001 study ${ }^{5}$ was a randomized, open-label study to compare the efficacy and safety of ABC/3TC FDC administered once daily to $\mathrm{ABC}$ (administered twice daily) and 3TC (administered once daily) given as separate entities, in combination with tenofovir (NRTI) and a new PI or NNRTI in antiretroviral-experienced adults experiencing VF defined as a VL $>1000$ copies/mL at the time of enrollment with 3 or fewer NRTI-associated mutations. The primary efficacy end point was the time-averaged change from baseline (average area under the curve minus baseline) in plasma HIV RNA over 48 weeks. A total of 186 subjects were enrolled. No significant differences were seen in the primary efficacy endpoint or in the percentage of participants who achieved an HIV RNA level $<50$ copies/mL. Tolerability was similar between the 2 groups.

Monotherapy with fixed dose combination of abacavir/ lamivudine/zidovudine (trizivir) is not considered standard of care for initial therapy due to inferior virologic efficacy, ${ }^{55}$ compared to a regimen containing a drug from another class (efavirenz).

\section{Safety, tolerability and adverse effects}

As previously discussed, neither ABC nor 3TC is metabolized by the CYP-450 system. Consequently, drug-drug interactions are limited. Nucleoside analogs are associated, by varying degrees, with mitochondrial dysfunction. This toxicity can result in dyslipidemia, lipoatrophy and lactic acidosis. Lactic acidosis results from the inhibition of mitochondrial DNA synthesis by the nucleoside analogs, resulting in anaerobic glycolysis and intracelluar accumulation of lactate. 3TC has greater affinity for mitochondrial DNA than does $\mathrm{ABC}{ }^{56,57}$ In vitro data have shown that neither $3 \mathrm{TC}$ nor $\mathrm{ABC}$ is associated with hepatic cytotoxicity or depletion of mitochondrial DNA. ${ }^{58}$ Furthermore, in a cohort study evaluating the risk factors for hyperlactatemia and lactic acidosis, ABC/3TC was associated with the lowest relative risk amongst NRTI pairs in the trial. ${ }^{59}$ The combination of $\mathrm{ABC}+3 \mathrm{TC}$ also has been shown to have minimal or no effects on in terms of lipoatrophy as well as favorable lipid insulin and other metabolic effects. ${ }^{60-62}$ Replacing stavudine with abacavir or zidovudine has been shown to improve stavudine-induced lipoatrophy. ${ }^{63}$

\section{Resistance}

ABC selects for mutations at positions 65 (K65R), 74 (L74V), $115(\mathrm{Y} 115 \mathrm{~F})$, and $184(\mathrm{M} 184 \mathrm{~V})$ in reverse transcriptase (RT). Lamivudine selects for mutations only at position 184 (M184/V/I) in RT. ${ }^{64}$ The M184V/I mutations confers substantial resistance to both $3 \mathrm{TC}$ and emtricitabine, but only about a 2 - to 3-fold increase in $\mathrm{IC}_{50}$ to abacavir. Thus, an isolated $\mathrm{M} 184 \mathrm{~V} / \mathrm{I}$ mutation does not result in complete loss of activity to abacavir. In addition, the M184V/I mutations may enhance susceptibility to zidovudine, stavudine, and tenofovir. ${ }^{64}$ Furthermore, the presence of the M184V/I mutation typically results in impaired viral replication capacity, which has been shown to decrease HIV RNA levels by about 0.5 logs. ${ }^{65,66}$ When $\mathrm{ABC}+3 \mathrm{TC}$ are used in combination with another class, the M184V mutation typically appears first and most frequently; the K65R mutation and L74V mutation are seen much less frequently. The abacavir-selected mutations K65R, L74V and Y115F each individually confer about three-to fourfold resistance to $\mathrm{ABC}$, but in combinations of 2 or 3 , they confer up to 8 - to 10 -fold resistance. ${ }^{67}$

The thymidine analogue mutations (TAMs), which include M41L, D67N, L210W and T215Y/F, K219Q/E/N, are not selected by $\mathrm{ABC}$ but rather by the thymidine analogues $\mathrm{ZDV}$ and $\mathrm{d} 4 \mathrm{~T}$. These mutations are associated with cross-resistance to all NRTIs, with increasing resistance as more of the TAMs accumulate. In isolation, they are associated with low-level resistance to $3 \mathrm{TC}$ and reduced virologic response to $\mathrm{ABC}$. Patients with four TAMs in combination with M184V and $\mathrm{L} 74 \mathrm{~V}$ have minimal virologic responses to $\mathrm{ABC}$-based regimens. ${ }^{68,69}$ Other mutations conferring high-level resistance to both ABC and 3TC in combination with TAMs are the T69 insertion mutation and the presence of E44D plus V1181. The Q151M complex also results in resistance to both drugs. ${ }^{70}$

\section{Conclusion and expert opinion}

$\mathrm{ABC} / 3 \mathrm{TC}$ is a well tolerated and effective NRTI backbone for both ARV-naive and ARV-experienced patients. The combination has been well studied and proven to 
be efficacious in multiple studies. The flexibility of using this NRTI backbone with other classes is an additional advantage. Resistance mutations have not limited the clinical utility of this combination when used with other classes. The availability of the drugs in both FDC and individual formulations gives physicians the additional flexibility of dose adjustment when required. Other advantages of this combination are the relative paucity of significant drugdrug interactions and the option to take the medication with or without food. However, all patients should be screened for HLA-B5701, prior to initiating treatment with $\mathrm{ABC}$ to prevent the occurrence of abacavir-hypersensitivity syndrome. Recent studies, albeit with conflicting results, have suggested a possible increased risk of myocardial infarction. One recent study showed a shortened duration to virologic failure in patients with pre-treatment HIV RNA $>100,000 \mathrm{~cm}^{3} / \mathrm{mL}$. Pending further studies, caution should be exercised in patients with underlying risk factors for CAD and baseline $\mathrm{VL}>100,000 \mathrm{~cm}^{3} / \mathrm{mL}$. In patients without these risk factors and a negative HLA-B5701 screen, the abacavir/lamivudine combination is a suitable alternative especially in patients with renal disease and contraindications to tenofovir.

\section{Disclosures}

RDM is on the Speakers' Bureau of ViiV Healthcare, and previously was a consultant to, and received grants from, GlaxoSmithKline.

\section{References}

1. DHHS Panel on Antiretroviral Guidelines for Adults and Adolescents -A Working Group of the Office of AIDS Research Advisory Council (OARAC). Guidelines for the use of antiretroviral agents in HIV-1 infected adults and adolescents. URL: http://AIDSinfo.nih.gov. Accessed Nov 3, 2008.

2. McDowell JA, Chittick GE, Stevens CP, et al. Pharmacokinetics of [(14C)] abacavir, a human immunodeficiency virus type-1 (HIV) reverse transcriptase inhibitor, administered in a single oral dose to HIV-1infected adults: a mass balance study. Antimicrob Agents Chemother. 1999;43:2855-2861.

3. McDowell JA, Lou Y, Symonds WS, Stein DS. Multiple-dose pharmacokinetics and pharmacodynamics of abacavir alone and in combination with zidovudine in human immunodeficiency virus-infected adults. Antimicrob Agents Chemother. 2000;44:2061-2067.

4. van Praag RM, van Heeswijk RP, Jurriaans S, et al. Penetration of the nucleoside analogue abacavir into the genital tract of men infected with human immunodeficiency virus Type 1. Clin Infect Dis. 2001;33:e91-e92.

5. Lamarca A, Clumeck N, Plettenberg A, et al. Efficacy and safety of a once-daily fixed-dose combination of abacavir/lamivudine compared with abacavir twice daily and lamivudine once daily as separate entities in antiretroviral-experienced HIV-1-infected patients (CAL 30001 Study). J Acquir Immune Defic Syndr. 2006;41:598-606.

6. Pilerio P. Shachoy-Clark AD, Para M, et al. A study examining the pharmacokinetics of abacavir and the intracellular carbovir triphosphate (GSK Protocol CAN10905). [abstract] Intersci Conf Antimicrob Agents Chemother. 2003;43 Sep 14-17: abstract A-1797.
7. Kewn S, Hoggard PG, Sales SD, et al. Development of enzymatic assays for quantification of intracellular lamivudine and carbovir triphosphate levels in peripheral blood mononuclear cells from human immunodeficiency virus-infected patients. Antimicrob Agents Chemother. 2002;46:135-43.

8. Harris M, Back D, Kewn S, Jutha S, Marina R, Montaner JS. Intracellular carbovir triphosphate levels in patients taking abacavir once a day. AIDS. 2002;16:1196-97.

9. Drusano GL, Bilello PA, Symonds WT, et al. Pharmacodynamics of abacavir in an in vitro hollow-fiber model system. Antimicrob Agents Chemother. 2002;46:464-470.

10. Moyle G, Boffito M, Fletcher C, et al. Steady-state pharmacokinetics of abacavir in plasma and intracellular carbovir triphosphate following administration of abacavir at 600 milligrams once daily and 300 milligrams twice daily in human immunodeficiency virus-infected subjects. J Antimicrob Chemother. 2009:1532-1538.

11. Keiser P, Nassar N, Skiest D, et al. Comparison of symptoms of influenza A with abacavir-associated hypersensitivity reaction. Int $J S T D \& A I D S$. 2003; $14: 478-481$.

12. Cutrell AG, Hernandez JE, Fleming JW, et al. Updated clinical risk factor analysis of suspected hypersensitivity reactions to abacavir. Ann Pharmacother. 2004;38:2171-2172.

13. Thurmond LM, Thorborn D, Ravitch J, et al. Immunohistologic characterization of skin rash observed in patients on abacavir who experience a hypersensitivity reaction. Antivir Ther. 2000;5(Suppl 5):53 (abstract P51).

14. Symonds W, Cutrell A, Edwards M, et al. Risk factor analysis of hypersensitivity reactions to abacavir. Clin Ther. 2002;24:565-573.

15. Walensky RP, Goldberg JH, Daily JP. Anaphylaxis after rechallenge with abacavir. AIDS. 1999;13:999-1000.

16. Easterbrook PJ, Waters A, Murad S, et al. Epidemiological risk factors for hypersensitivity reactions to abacavir. HIV Med. 2003;4:321-324.

17. Loeliger AE, Steel H, McGuirk S, Powell WS, Hetherington SV. The abacavir hypersensitivity reaction and interruptions in therapy. AIDS. 2001;15:1325-1326.

18. EPZICOM ${ }^{\mathrm{TM}}$ (abacavir sulfate and lamivudine) tablets [product information]. Research triangle Park, North Carolina: GlaxoSmithKline, March 2006.

19. Hetherington S, McGuirk S, Powell G, et al. Hypersensitivity reactions during therapy with the nucleoside reverse transcriptase inhibitor abacavir. Clin Ther. 2001;23:1603-1614.

20. Dargere S, Verdon R, Bouhier K, Bazin C. Disseminated intravascular coagulation as a manifestation of abacavir hypersensitivity reaction. AIDS. 2002;16:1696-1697.

21. Bourezane Y, Salard D, Hoen B, Vandel S, Drobacheff C, Laurent R. DRESS (drug rash with eosinophilia and systemic symptoms) syndrome associated with nevirapine therapy. Clin Infect Dis. 1998;27: 1321-1322.

22. Bossi P, Colin D, Bricaire F, Caumes E. Hypersensitivity syndrome associated with efavirenz therapy. Clin Infect Dis. 2000;30:227-228.

23. Ryan C, Madalon M, Wortham DW, Graziano FM. Sulfa hypersensitivity in patients with HIV infection: onset, treatment, critical review of the literature. WMJ. 1998;97:23-27.

24. Wolf CR, Smith G, Smith RL. Science, medicine, and the future: Pharmacogenetics. BMJ. 2000;320:987-990.

25. Koopmans PP, van der Ven AL, Vree TB, van der Meer JW. Pathogenesis of hypersensitivity reactions to drugs in patients with HIV infection: allergic or toxic? AIDS. 1995;9:217-222.

26. Peyrieere H, Nicolas J, Siffert M, Demoly P, Hillaire-Buys D, Reynes J. Hypersensitivity related to abacavir in two members of a family. Ann Pharmacother. 2001;35:1291-1292.

27. Hetherington S, Hughes AR, Mosteller M, et al. Genetic variations in HLA-B region and hypersensitivity reactions to abacavir. Lancet. 2002;359:1121-1122.

28. Mallal S, Nolan D, Witt C, et al. Association between presence of HLA-B*5701, HLA-DR7, and HLA-DQ3 and hypersensitivity to HIV-1 reverse-transcriptase inhibitor abacavir. Lancet. 2002;359:727-732. 
29. Martin AM, Nolan D, Gaudieri S, et al. Predisposition to abacavir hypersensitivity conferred by HLA-B*5701 and a haplotypic Hsp70-Hom variant. Proc Natl Acad Sci U S A. 2004;101:4180-4185.

30. Rauch A, Nolan D, Martin A, McKinnon E, Almeida C, Mallal S. Prospective genetic screening decreases the incidence of abacavir hypersensitivity reactions in the Western Australian HIV cohort study. Clin Infect Dis. 2006;43:99-102.

31. Reeves I, Churchill D, Fisher M, et al. Screening for HLA-B*5701 reduces the frequency of abacavir hypersensitivity reactions. Antivir Ther. 2006;11 (Suppl 3):L11 (abstract 14).

32. Mallal S, Phillips E, Carosi G, et al. HLA-B*5701 Screening for hypersensitivity to abacavir. $N$ Engl J Med. 2008;358:568-579.

33. Saag M, Balu R, Phillips E, et al. High sensitivity of human leukocyte antigen-b*5701 as a marker for immunologically confirmed abacavir hypersensitivity in white and black patients. Clin Infect Dis. 2008. 46:1111-1118

34. Wit FW, Wood R, Horban A, et al. Prednisone does not prevent hypersensitivity reactions in antiretroviral drug regimens containing abacavir with or without nevirapine. AIDS. 2001;15:2423-2429.

35. DAD Study Group* Use of nucleoside reverse transcriptase inhibitors and risk of myocardial infarction in HIV-infected patients enrolled in the DAD study: a multi-cohort collaboration. Lancet. 2008;371: 1417-1426.

36. Strategies for Management of Anti-Retroviral Therapy/INSIGHT; DAD Study Groups. Use of nucleoside reverse transcriptase inhibitors and risk of myocardial infarction in HIV-infected patients. AIDS. 2008;22: F17-F24.

37. Brothers $\mathrm{CH}$, Hernandez JE, Cutrell AG, et al. Risk of myocardial infarction and abacavir therapy: no increased risk across 52 GlaxoSmithKlinesponsored clinical trials in adult subjects. J Acquir Immune Defic Syndr. 2009;51:20-28.

38. Bedimo R, Westfall A, Drechsler H, Tebas P. Abacavir use and risk of acute myocardial infarction and cerebrovascular disease in the HAART era. 5th IAS Conference on HIV Pathogenesis, Treatment and Prevention; July 19-22, 2009. Cape Town. Abstract MOAB202.

39. Benson CA, Ribaudo H, Zheng E, et al. No Association of Abacavir (ABC) Use with Risk of Myocardial Infarction (MI) or Severe Cardiovascular Disease Events (SCVD): Results from ACTG A5001/ALLRT. 16th CROI Conference on Retroviruses and Opportunistic Infections Montreal, Canada; February 8-11, 2009.

40. Bruno R, Regazzi MB, Ciappina V, et al. Comparison of the plasma pharmacokinetics of lamivudine during twice and once daily administration in patients with HIV. Clin Pharmacokinet. 2001; 40:695-700.

41. Moore KH, Barrett JE, Shaw S, et al. The pharmacokinetics of lamivudine phosphorylation in peripheral blood mononuclear cells from patients infected with HIV-1. AIDS. 1999;13:2239-2250.

42. Yuen GJ, Lou Y, Bumgamer NF, et al. Equivalent steady-state pharmacokinetics of lamivudine in plasma and lamivudine triphosphate within cells following administration of lamivudine at 300 milligrams once daily and 150 milligrams twice daily. Antimicrob Agents Chemother. 2004;48:176-182.

43. Moore KH, Yuen GJ, Raasch RH, et al. Pharmacokinetics of lamivudine administered alone and with trimethoprim-sulfamethoxazole. Clin Pharmacol Ther. 1996;59:550-558.

44. McDowell JA, Chittick GE, Stevens CP, Edwards KD, Stein DS. Pharmacokinetic interaction of abacavir (1592U89) and ethanol in human immunodeficiency virus-infected adults. Antimicrob Agents Chemother. 2000;44:1686-1690

45. Dejesus E, Herrera G, Teofilo E, et al. Abacavir versus zidovudine combined with lamivudine and efavirenz, for the treatment of antiretroviral-naïve HIV-infected adults. Cin Infect Dis. 2004;39:1038-1046.

46. MacArthur RD, Chen L, Peng G, et al. Efficacy and Safety of Abacavir Plus Lamivudine Versus Didanosine Plus Stavudine When Combined with a Protease Inhibitor, a Nonnucleoside Reverse Transcriptase Inhibitor, or Both in HIV-1 Positive Antiretroviral-Naive Persons. HIV Clin Trials. 2004;5:361-370.
47. Sax P, Tierney C, Collier A, et al. ACTG 5202: Shorter time to virologic failure with $\mathrm{ABC} / 3 \mathrm{TC}$ than tenofovir/FTC in treatment-naive subjects with HIV RNA $>100,000.17$ th International AIDS Conference; Aug 3-8, 2008; Mexico City, Mexico. Abstract THAB0303.

48. Smith KY, Patel P, Fine D, et al. Randomized, double-blind, placebo-matched, multicenter trial of abacavir/lamivudine or tenofovir/ emtricitabine with lopinavir/ritonavir for initial HIV treatment. AIDS. 2009;23:1547-1556.

49. Rodriguez-French A, Boghossian J, Gray GE, et al. The NEAT study: a 48-week open-label study to compare the antiviral efficacy and safety of GW433908 versus nelfinavir in antiretroviral therapy-naïve HIV-1infected patients. J Acquir Immune Defic Syndr. 2004;35:22-32.

50. Gathe JC Jr, Ive P, Wood R, et al. SOLO: 48-week efficacy and safety comparison of once-daily fosamprenavir/ritonavir versus twicedaily nelfinavir in naïve HIV-1-infected patients. AIDS. 2004;18: 1529-1537.

51. Moyle GJ, Dejesus E, Cahn P, et al. Abacavir once or twice daily combined with once-daily lamivudine and efavirenz for the treatment of antiretroviral-naïve HIV-infected adults: results of the Ziagen Once Daily in Antiretroviral Combination Study. J Acquir Immune Defic Syndr. 2005;38:417-425.

52. Bartlett JA, Johnson J, Herrera G, et al. Long term-results of initial therapy with abacavir and Lamivudine combined with Efavirenz, Amprenavir/Ritonavir, or Stavudine. J Acquir Immune Defic Syndr. 2006;43:284-292.

53. Eron J Jr, Yeni P, Gathe J Jr, et al. The KLEAN study of fosamprenavirritonavir versus lopinavir-ritonavir, each in combination with abacavirlamivudine, for initial treatment of HIV infection over 48 weeks: a randomised non-inferiority trial. Lancet. 2006;368:476-482.

54. Sosa N, Hill-Zabala C, Dejesus E, et al. Abacavir and lamivudine fixed-dose combination tablet once-daily compared with abacavir and lamivudine twice-daily in HIV-1 infected patients over 48 weeks (ESS30008, SEAL). J Acquir Immune Defic Syndr. 2005;40: 422-427.

55. Gulick R, Ribaudo H, Shikuma C, et al. Triple-nucleoside regimens versus efavirenz-containing regimens for the initial treatment of HIV-1 infection. N Engl J Med. 2004;350:1850-1861.

56. Johnson AA, Ray AS, Hanes J, et al. Toxicity of antiviral nucleoside analogs and the human mitochondrial DNA polymerase. J Biol Chem. 2001;276:40847-40857.

57. Kakuda TN. Pharmacology of nucleoside and nucleotide reverse transcriptase inhibitor-induced mitochondrial toxicity. Clin Ther. 2000;22:685-708.

58. Birkus G, Hitchcock MJ, Cihlar T. Assessment of mitochondrial toxicity in human cells treated with tenofovir: comparison with other nucleoside reverse transcriptase inhibitors. Antimicrob Agents Chemother. 2002;46:716-723.

59. Moyle GJ, Datta D, Mandalia S, Morlese J, Asboe D, Gazzard BG. Hyperlactaemia and lactic acidosis during antiretroviral therapy: relevance, reproducibility and possible risk factors. AIDS. 2002;16: 1341-1349.

60. Podzamczer D, Ferrer E, Sanchez P, et al. Less lipoatrophy and better lipid profile with abacavir as compared to stavudine: 96-week results of a randomized study. J Acquir Immune Defic Syndr. 2007;44: 139-147.

61. Carr A, Workman C, Smith DE, et al. Abacavir substitution for nucleoside analogs in patients with HIV lipoatrophy: randomized trial. JAMA. 2002;288:207-215.

62. Shlay J, Visnegarwala F, Bartsch G, et al. Body composition and metabolic changes in antiretroviral-naïve patients randomized to didanosine and stavudine vs abacavir and lamivudine. J Acquir Immune Defic Syndr. 2005;38:147-155.

63. McComsey G, Ward D, Hessenthaler S, et al. Lipoatrophy improvement following switch to ABC or AZT from D4T, Tarheel study. Clin Infec Dis. 2004;38:263-270.

64. D’Aquila RT, Schapiro JM, Brun-Vezinet F, et al. Drug resistance mutations in HIV-1. Top HIV Med. 2002;10:21-25. 
65. Eron JJ, Benoit SL, Jemsek J, et al. Treatment with lamivudine, zidovudine, or both in HIV-positive patients with 200 to $500 \mathrm{CD} 4+$ cells per cubic millimeter. North American HIV Working Party. N Engl J Med. 1995;333:1662-1669.

66. Frankel FA, Invernizzi CF, Oliveira M, Wainberg MA. Diminished efficiency of HIV-1 reverse transcriptase containing the K65R and M184V drug resistance mutations. AIDS. 2007;21:665-675.

67. Tisdale M, Alnadaf T, Cousens D. Combination of mutations in human immunodeficiency virus Type 1 reverse transcriptase required for resistance to the carbocyclic nucleoside 1592U89. Antimicrob Agents Chemother. 1997;41:1094-1098.
68. Brun-Vezinet F, Deschamps, Ruffault A, et al. Clinically relevant interpretation of genotype for resistance to abacavir. AIDS. 2003;17:1795-1802.

69. Walter H, Schmidt B, Wervein M, et al. Prediction of abacavir resistance from genotypic data: impact of zidovudine and lamivudine resistance in vitro and in vivo. Antimicrob Agents Chemother. 2002;46:89-94.

70. Van Laethem K, Witvrouw M, Balzarini J, et al. Patient HIV-1 strains carrying the multiple nucleoside resistance mutations are cross-resistant to abacavir. AIDS. 2000;14:469-471.

\section{Publish your work in this journal}

Therapeutics and Clinical Risk Management is an international, peerreviewed journal of clinical therapeutics and risk management, focusing on concise rapid reporting of clinical studies in all therapeutic areas, outcomes, safety, and programs for the effective, safe, and sustained use of medicines. This journal is indexed on PubMed Central, CAS,
EMBase, Scopus and the Elsevier Bibliographic databases. The manuscript management system is completely online and includes a very quick and fair peer-review system, which is all easy to use. Visit http://www.dovepress.com/testimonials.php to read real quotes from published authors.

Submit your manuscript here: http://www.dovepress.com/therapeutics-and-clinical-risk-management-journal 